\section{MS27-P2 Indexing electron diffraction patterns from randomly-oriented crystals}

Yunchen Wang ${ }^{1}$, Wei Wan ${ }^{1}$, Xiaodong Zou ${ }^{1}$

1. Berzelii Center EXSELENT on Porous Materials and Inorganic and Structural Chemistry, Department of Materials and Environmental Chemistry, Stockholm University, SE-106 91 Stockholm

email: yunchen.wang@mmk.su.se

Electron diffraction (ED) can be used to study nanometre-sized crystals and provides information about the unit cell, space group and even intensities for a complete structure solution. With a known unit cell, ED patterns can be indexed. The reflection indices are found after indexing and the reflection conditions can be used to derive the space group. Indexing is usually done with in-zone ED patterns from aligned crystals. Alignment of the crystal can be time consuming and for many electron beam sensitive materials it is very difficult, even impossible. Indexing ED patterns from randomly orientated crystals is thus necessary and it gives valuable information about the phase of the material and the space group. The orientation of the crystal can be determined and quantitative intensities of the reflections extracted for structure solution. Similar problem has been studied in femtosecond X-ray crystallography using X-ray free electron laser [1]. However the algorithms cannot be adopted for electron data due to the differences in wavelengths. Here we propose a fast and robust approach for indexing ED patterns from randomly orientated crystals, given the unit cell parameters. Rather than measuring the $\mathrm{d}^{*}$ values and mutual angles of the reflection vectors and matching them to the calculated values, we use instead the "difference vectors", which are obtained by subtracting the 2D coordinates of pairs of diffraction spots. Difference vectors typically have shorter $d^{*}$ values than the original reflection vectors and are thus easier to index (less ambiguity). Zone axes given by the difference vectors are close to the actual zone axes along which the ED patterns are taken. They can be used to quickly narrow down the solution for indexing all the reflections in the ED pattern. The algorithm has been tested with the frames of a $3 \mathrm{D}$ ED dataset from zeolite silicalite-1 (unit cell: $a=20.090 \AA, b=19,738 \AA, c=$ 13,142 Å, space group: Pnma).

[1] H. N. Chapman, et al., Nature 470, 73(2011)

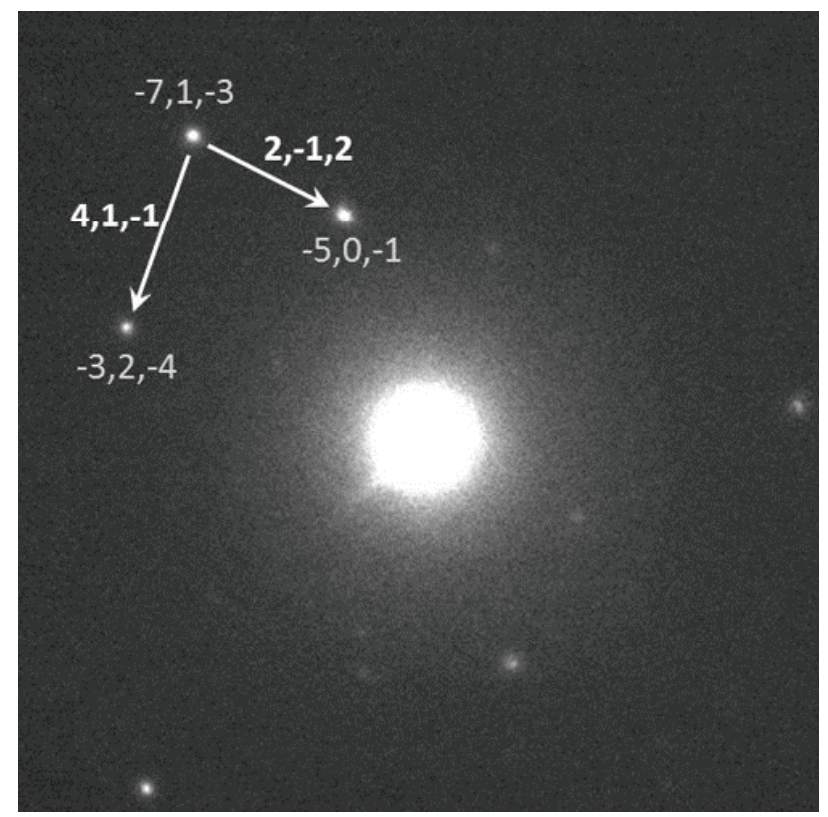

Figure 1. Illustration of the "difference vector" approach for indexing ED patterns from randomly orientated crystals. The ED pattern was taken from a zeolite silicalite-1 crystal in a random orientation. Three reflections generate difference vectors which are of lower resolution and easier to index.

Keywords: electron diffraction, indexing, random, crystallography 